\title{
Small Angle X-ray Scattering Study of Magnetic Nanofluid Exposed to an Electric Field
}

\author{
M. Rajnak ${ }^{a, b, *}$, V.M. Garamus ${ }^{c}$, M. Timko $^{a}$, P. Kopcansky $^{a}$, K. Paulovicova $^{a}$, \\ J. Kurimsky ${ }^{b}$, B. DolniK ${ }^{b}$ And R. Cimbala ${ }^{b}$ \\ ${ }^{a}$ Institute of Experimental Physics, SAS, Watsonova 47, 04001 Košice, Slovakia \\ ${ }^{b}$ Faculty of Electrical Engineering and Informatics, Technical University of Košice, Letná 9, 04200 Košice, Slovakia \\ ${ }^{c}$ Helmholtz-Zentrum Geesthacht, Centre for Materials and Coastal Research, \\ Max-Planck-Str., 21502 Geesthacht, Germany
}

\begin{abstract}
We report on the investigation of a transformer oil-based magnetic nanofluid exposed to an electric field by means of synchrotron small angle X-ray scattering. Two types of small angle X-ray scattering experiments were carried out. In the first one, the electric field up to $6 \mathrm{kV} / \mathrm{cm}$ was generated in the nanofluid between two immersed electrodes. The other experiment focused on the nanofluid in an external electric field up to $10 \mathrm{kV} / \mathrm{cm}$, when the electrodes were not in a direct electrical contact with the nanofluid. In the available range $\left(0.02-4.5 \mathrm{~nm}^{-1}\right)$ of scattering vector $q$, the non-contact mode has no effect on the scattering intensity. The contact mode yielded noticeable low- $q$ intensity variations. In comparison to small angle neutron scattering, the small angle X-ray scattering study did not prove the proportional increase in the low $q$ scattering intensity with increasing electric field, but rather stochastic variations. The observed intensity variations reflect the local structural nanofluid changes caused by the induced electrohydrodynamics. The electrical conductivity and relaxation processes are pointed out as favorable conditions for electrohydrodynamics in the magnetic nanofluid.
\end{abstract}

DOI: 10.12693/APhysPolA.137.942

PACS/topics: magnetic nanofluid, small angle X-ray scattering (SAXS), electric field

\section{Introduction}

One of the most intensively studied effects in the physics of magnetic nanofluids (MNF) is the formation of anisotropic magnetic nanoparticle (MNP) aggregates. Their behavior can be controlled by external magnetic fields. MNF spatial structure induced by the resulting magnetic field is often studied by the method of small angle neutron scattering (SANS) [1,2]. Recently, a SANS study revealed that similar MNP aggregates can be formed in a transformer oil-based MNF exposed to an external electric field [3]. This phenomenon has been described as a result of the effective MNP polarization and subsequent electric dipole-dipole interaction. In the field of rheological properties, which are widely studied for various materials $[4,5]$, it was found that the viscosity of MNF increases with increasing electric field intensity [6]. The effect is more pronounced at low shear rates, which indicates the competitive nature of the electric and flow forces on the MNP aggregation. Thus, the knowledge of MNP aggregation and spatial structure of MNF in an electric field is essential for potential applications in electrical engineering. In SANS experiment, the typical size of the irradiated sample area is of the order of $1 \mathrm{~cm}^{2}$, yielding the average structure of the irradiated MNF. Owing to the long measurement time (at least $10 \mathrm{~min}$, as measured in [3]), one cannot obtain in-

\footnotetext{
*corresponding author; e-mail: rajnak@saske.sk
}

formation about possible kinetics in the MNF by SANS. Especially, when an electric field induces low current in the MNF, the electrohydrodynamic motion can significantly influence the MNP aggregate formation. To focus on a local MNF structure (smaller irradiated area) and shorten the measuring time, high brilliance X-ray beam should be used and small angle X-ray scattering (SAXS) studies to complement the SANS experiment. In research on MNF, the SAXS is often applied due to the invisibility of surfactant around MNP, when magnetic cores are considered as quasi-spherical particles. Moreover, in SAXS there is no magnetic scattering contribution like in SANS, which also becomes anisotropic under magnetic fields [7]. To account for the core-shell structure of the MNP, the SAXS data can be interpreted according to a pearl necklace model [8].

In this paper we report the investigation of a transformer oil-based MNF exposed to an electric field by means of SAXS using a high brilliance synchrotron radiation. To verify the possible electrohydrodynamics, two types of experiments are performed, when the electrodes are applied internally and externally with regard to the MNF.

\section{Materials and methods}

In the investigated MNF, a commercial transformer oil is used as a base liquid. The dispersive phase consists of iron oxide MNP (mean diameter of $10 \mathrm{~nm}$ ) synthesized by a chemical co-precipitation in a well proven way [9]. The MNP is stabilized with a single layer of oleic acid. The magnetic volume fraction was estimated 


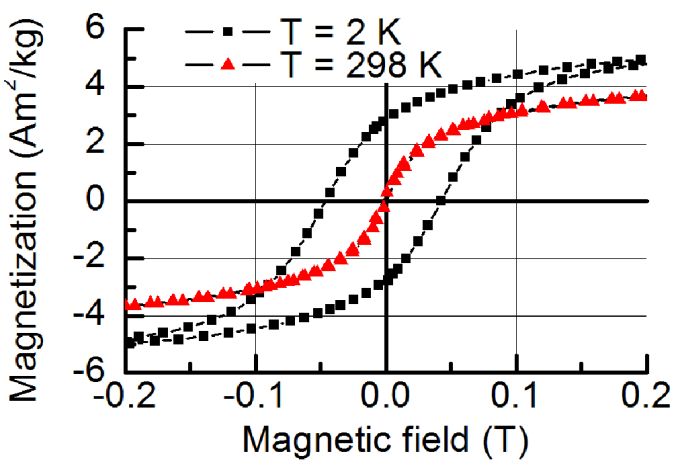

Fig. 1. Specific magnetization of the magnetic nanofluid measured at the temperature of $2 \mathrm{~K}$ and $298 \mathrm{~K}$.

as a ratio of the MNF volume magnetization of saturation $(4.9 \mathrm{kA} / \mathrm{m})$ to the magnetization of saturation of magnetite $(446 \mathrm{kA} / \mathrm{m})[10]$, yielding the value of about $1.1 \%$. The magnetization was measured by a vibrating sample magnetometer installed on a cryogen free superconducting magnet from Cryogenic Ltd. In Fig. 1, the obtained hysteresis loops are presented. The graph shows zero coercivity at room temperature, indicating the superparamagnetic state with the absence of MNP interactions. The blocked state is reflected in the pronounced hysteresis measured at $2 \mathrm{~K}$.

The SAXS experiments were conducted at P12 BioSAXS beam-line (EMBL) utilizing the high brilliance 3rd generation synchrotron radiation source PETRA III at DESY, Hamburg, Germany. The incident beam (of the wavelength of $0.66 \AA$ ) with the aperture of 0.1 by $0.2 \mathrm{~mm}$ was used. The silver behenate was utilized to calibrate the scattering vector interval. Two sample holders with electrodes were prepared. The first one consists of a glass capillary (1 $\mathrm{mm}$ in diameter), to which a pair of plane electrodes is attached from the outside $(4 \mathrm{~mm}$ from each other). This setup constitutes the non-contact mode with the electrodes electrically insulated from the sample. The other sample holder consists of a glass cuvette $(1 \mathrm{~mm}$ thick) with two tubular electrodes inserted $1 \mathrm{~cm}$ each from other in the cuvette (as shown in [3]). In this case, the sample is in direct electrical contact with the stainless steel electrodes. To power the electrodes, DC voltage was applied up to $6 \mathrm{kV}$ for the contact mode and up to $4 \mathrm{kV}$ for the non-contact mode. The SAXS measurements ( 5 frames per second, room temperature) started a few seconds after the application of the voltage. Then, 1D scattering intensities normalized to transmitted beam were obtained by automatic procedure with checking of possible radiation damage [11].

\section{Results and discussion}

The SAXS experimental results are presented as dependent of the scattering intensity $I$ on the scattering vector $q$, which is usually given by

$$
I(q)=\varphi P(q) S(q)+\mathrm{bkg},
$$

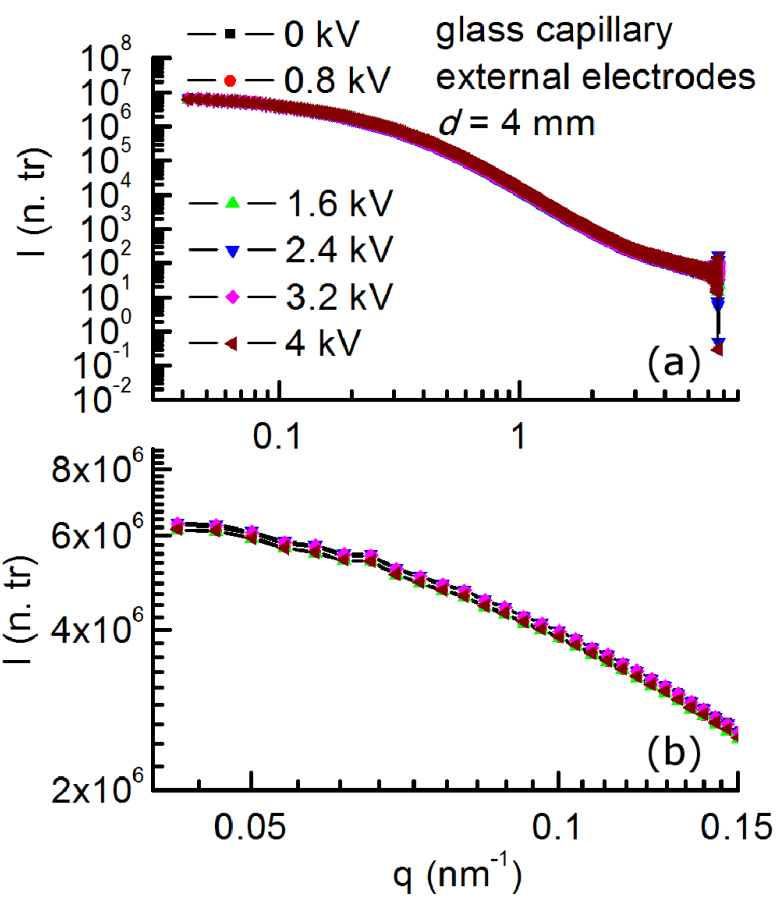

Fig. 2. Experimental SAXS curves for the sample in the capillary (non-contact mode) under various voltage levels (a). The zoomed view in the low $q$ region (b).

where $\varphi$ is the particle volume fraction, $P(q)$ is the scattering of single particle, $S(q)$ is the interparticle structure factor accounting for the interparticle correlations, and bkg is the incoherent background [8]. In Fig. 2, one can see 1D SAXS curves measured on the MNF in the capillary with the external electrodes powered from $0 \mathrm{kV}$ up to $4 \mathrm{kV}$. The curves in Fig. 2a exhibit no fringes typical for particle form factors, thus indicating that the smearing is due to the MNP polydispersity. Moreover, the obtained profiles match with each other in the available $q$ range. This signifies no effect of the external electric field on the MNF structure. As the electric field between the electrodes is quasi-homogeneous, the MNP does not experience dielectrophoresis, even though the polarization of MNP may be effective due to the different permittivity between the MNP and the surrounding oil. Thus, owing to the absence of dielectrophoresis and electrohydrodynamic flow (negligibly small current), the MNF remains in the initial equilibrium state. This can be concluded from the zoomed view on the low $q$ scattering region in Fig. 2b, where the tiny changes in SAXS profiles are within the experimental error.

Different behavior was observed in the cuvette with the electrodes immersed in the MNF. The SAXS results obtained in this contact mode are presented in Fig. 3. Especially in the lower $q$ region, the scattering profiles exhibit variations in the amplitude of the scattering intensity under various voltages, as seen from Fig. 3b. However, the variations do not follow the increasing voltage. Instead, stochastic changes in the scattering intensity level are observed. This behavior reflects stochastic variations 


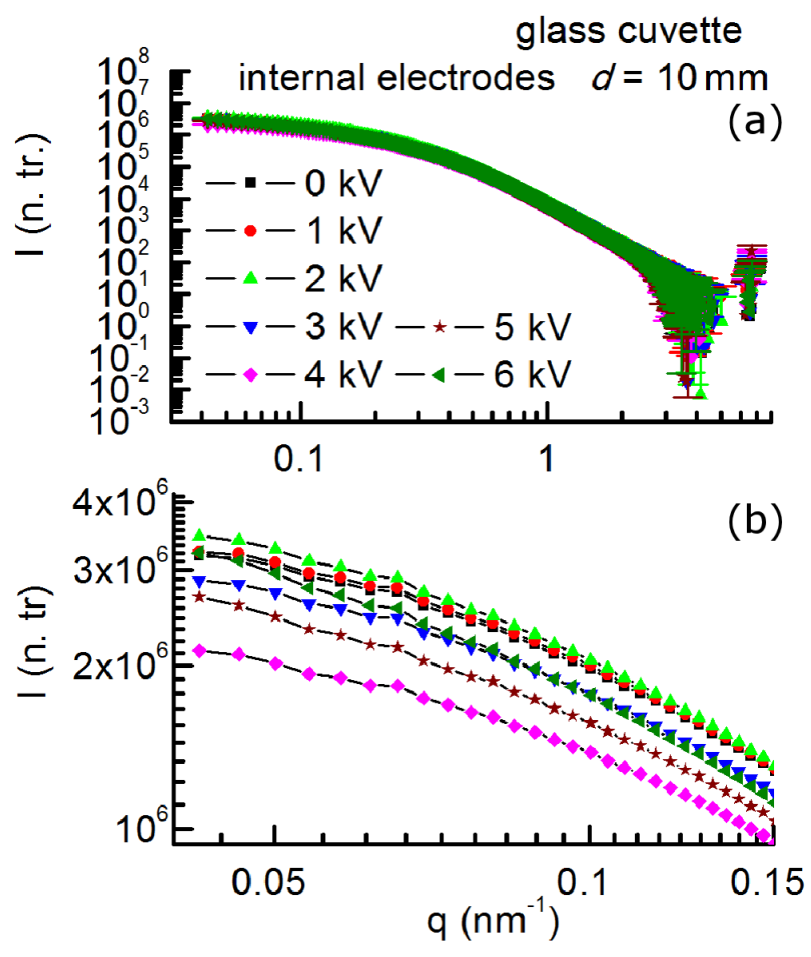

Fig. 3. Experimental SAXS curves for the sample in the cuvette (contact mode) under various voltage levels (a). The zoomed view in the low $q$ region (b).

of the MNF concentration in the irradiated sample volume, as the scattering intensity is directly proportional to the MNP volume fraction (1). The observed concentration variations can be interpreted as a result of the induced electrohydrodynamics. This well-known phenomenon arises when the electric field acts on the free charge (residual ions in the MNF) and particles, giving rise to a force on the fluid and therefore flow. As the electrodes are in contact with the MNF, the current is maintained and one can measure tens of $\mathrm{nA}$, as shown in [3]. On the other hand, the migrating particles experience the polarizing forces and undergo the electric interparticle forces (magnetic interactions are neglected, as concluded from the magnetization curve in Fig. 1), forming more concentrated regions in the MNF. If the concentrated region is electrohydrodynamically brought in the irradiated sample volume, the increase in the scattering intensity is detected. The favorable conditions for MNF electrohydrodynamics in the DC electric field, due to slow polarization processes and conduction mechanisms, are confirmed in another study [12].

\section{Conclusions}

In this paper, SAXS experimental study on transformer oil-based magnetic nanofluid in electric field was carried out. The scattering profiles are compared for the magnetic nanofluid in direct electrical contact with the electrodes and externally exposed to the electric field.
No scattering changes were observed for the non-contact mode, reflecting the unchanged initial structure of the nanofluid in the electric field. On the other hand, the stochastic variation in the scattering intensity under various voltages was detected in the direct contact mode. This is a result of the small size X-ray beam (small irradiated area) scattered from the flowing concentration inhomogeneity. The inhomogeneity is brought about by the conductivity and polarization of the particles and the resulted electrohydrodynamics.

\section{Acknowledgments}

This work was supported by the Slovak Academy of Sciences and Ministry of Education in the framework of projects VEGA 2/0011/20, and 1/0340/18, Ministry of Education Agency for structural funds of EU: ITMS 26220220186 and ITMS 26220120055, COST CA15119 NANOUPTAKE, Slovak Research and Development Agency APVV-18-0160, APVV-15-0438, and APVV-0460-12. Dr. D.C.F. Wieland (HZG) is acknowledged for helping with the preparation of the high voltage SAXS sample holder.

\section{References}

[1] M.V. Avdeev, V.L. Aksenov, Phys.-Usp. 53, 971 (2011).

[2] S. Mühlbauer, D. Honecker, É.A. Périgo, et al., Rev. Mod. Phys. 91, 015004 (2019).

[3] M. Rajnak, V.I. Petrenko, M.V. Avdeev, O.I. Ivankov, A. Feoktystov, B. Dolnik, J. Kurimsky, P. Kopcansky, M. Timko, Appl. Phys. Lett. 107, 073108 (2015).

[4] A. Pietrikova, M. Kravcik, in: 35th Int. Spring Seminar on Electronics Technology, IEEE, 2012, p. 138.

[5] A. Pietrikova, M. Kravcik, in: Proc. 2011 34th Int. Spring Seminar on Electronics Technology, IEEE, 2011, p. 94.

[6] M. Rajnak, M. Timko, P. Kopcansky, et al., J. Magn. Magn. Mater. 431, 99 (2017).

[7] A.A. Veligzhanin, D.I. Frey, A.V. Shulenina, A.Y. Gruzinov, Y.V. Zubavichus, M.V. Avdeev, J. Magn. Magn. Mater. 459, 285 (2018).

[8] M. Bonini, E. Fratini, P. Baglioni, Mater. Sci. Eng. C 27, 1377 (2007)

[9] L. Vékás, D. Bica, M.V. Avdeev, China Particuology 5, 43 (2007).

[10] R.E. Rosensweig, Ferrohydrodynamics, Dover Publ., Mineola (NY) 2013.

[11] C.E. Blanchet, A. Spilotros, F. Schwemmer, et al., J. Appl. Crystallogr. 48, 431 (2015).

[12] M. Rajnak, B. Dolnik, J. Kurimsky, R. Cimbala, P. Kopcansky, M. Timko, J. Chem. Phys. 146, 014704 (2017). 